\title{
Drug pipeline 3Q17
}

\section{Laura DeFrancesco}

Drug approvals are way up through three quarters, including a number of first-in-class approvals. The first drug targeting a metabolic enzyme mutated in cancer (Celgene's mitochondrial isocitrate dehydrogenase) was approved. The approval of Novartis' highly anticipated CAR-T cancer therapy Kymriah for acute

\section{Historic US regulatory approvals by lead indication}

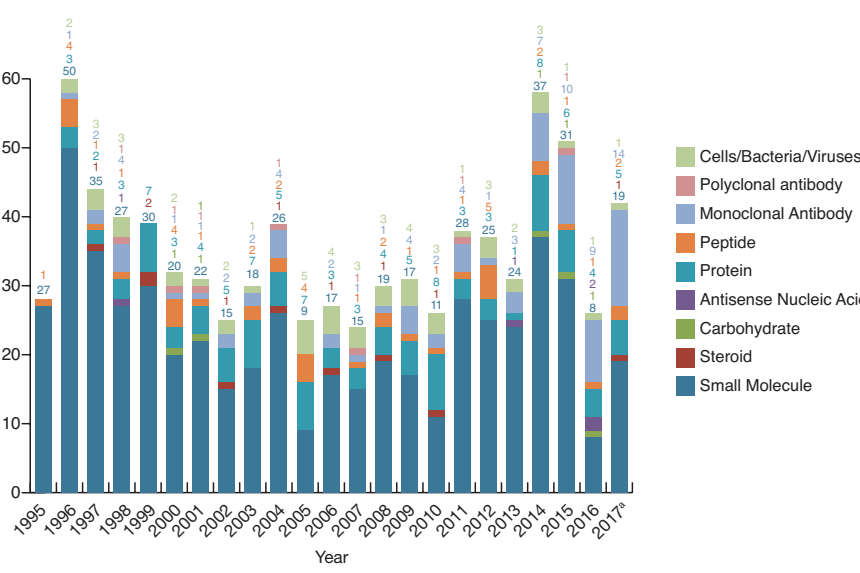

a2017 partial year ending September 30. Source: BioMedTracker a service of Sagient Research (http://www.biomedtracker.com)

Notable regulatory approvals (3Q17)

\begin{tabular}{ll} 
Drug/company & Indication \\
\hline Aliqopa (copanlisib)/ & Indolent non-Hodgkin
\end{tabular}

Bayer lymphoma

Tremfya (guselkumab)/ Psoriasis

J\&J

Nerlynx (neratinib male- Breast cancer ate)/Puma

(a)

9/14/2017 FDA granted accelerated approval for this small-molecule PI3K/AKT pathway inhibitor 7/13/2017 FDA approved this fully human monoclonal antibody targeting only the p19 subunit of IL-23

7/17/2017 FDA approved this small-molecule HER-2 inhibitor for women previously treated with Herceptin (trastuzumab) to prevent recurrence

\begin{tabular}{lll}
\hline $\begin{array}{l}\text { Idhifa (enasidenib)/ } \\
\text { Celgene }\end{array}$ & $\begin{array}{l}\text { Acute myelogenous } \\
\text { leukemia }\end{array}$ & $\begin{array}{l}\text { 8/1/2017 FDA approves this small-molecule } \\
\text { first-in-class inhibitor of mitochondrial isocitrate } \\
\text { dehydrogenase }\end{array}$ \\
\hline $\begin{array}{l}\text { Besponsa (inotuzumab } \\
\text { ozogamicin)/Pfizer }\end{array}$ & $\begin{array}{l}\text { Acute lymphocytic leu- } \\
\text { kemia }\end{array}$ & $\begin{array}{l}\text { 8/17/2017 FDA approves this humanized IgG4 } \\
\text { monoclonal antibody against CD22 conjugated } \\
\text { with N-acetyl gamma calicheamicin }\end{array}$ \\
\hline $\begin{array}{l}\text { Mylotarg (gemtuzumab } \\
\text { ozogamicin)/Pfizer }\end{array}$ & $\begin{array}{l}\text { Acute myelogenous } \\
\text { leukemia }\end{array}$ & $\begin{array}{l}9 / 1 / 2017 \text { FDA approves this second try at } \\
\text { humanized monoclonal lgG4 antibody against } \\
\text { CD33 conjugated with calicheamicin }\end{array}$ \\
\hline $\begin{array}{l}\text { Kymriah (tisagenlecleu- } \\
\text { cel-t)/Novartis }\end{array}$ & $\begin{array}{l}\text { Acute lymphocytic leu- } \\
\text { kemia }\end{array}$ & $\begin{array}{l}\text { 8/30/2017 FDA approved first-in-class CAR-T } \\
\text { cells against CD-19 bearing cells }\end{array}$ \\
\hline Verzenio (abemaciclib)/ & Breast cancer & $\begin{array}{l}9 / 28 / 2017 \text { FDA approves this small-molecule } \\
\text { cyclin-dependent kinase (CDK)4 and CDK6 } \\
\text { inhibitor }\end{array}$ \\
Eli Lilly & &
\end{tabular}

\section{Breakthrough drug designation}

\begin{tabular}{|c|c|c|}
\hline $\begin{array}{l}\text { Venclexta (venetoclax)/ } \\
\text { AbbVie }\end{array}$ & $\begin{array}{l}\text { Acute myelogenous } \\
\text { leukemia }\end{array}$ & Small-molecule Bcl-2 selective inhibitor \\
\hline $\begin{array}{l}\text { Imfinzi (durvalumab)/ } \\
\text { AstraZeneca }\end{array}$ & Non-small cell lung cancer & $\begin{array}{l}\text { Human IgG1к monoclonal antibody directed } \\
\text { against B7-H1 T-cell activator }\end{array}$ \\
\hline $\begin{array}{l}\text { Mogamulizumab/Kyowa } \\
\text { Hakko Kirin }\end{array}$ & $\begin{array}{l}\text { Cutaneous T-cell lym- } \\
\text { phoma }\end{array}$ & $\begin{array}{l}\text { Humanized monoclonal antibody for CCR4 } \\
\text { chemokine receptors selectively expressed in } \\
\text { T-helper type } 2 \text { (Th2) cells }\end{array}$ \\
\hline $\begin{array}{l}\text { DS-8201/Daiichi } \\
\text { Sankyo }\end{array}$ & Breast cancer & $\begin{array}{l}\text { Humanized anti-HER2 monoclonal antibody con- } \\
\text { jugated to exatecan derivative }\end{array}$ \\
\hline $\begin{array}{l}\text { EB-101/Abeona } \\
\text { Therapeutics }\end{array}$ & Epidermolysis bullosa & $\begin{array}{l}\text { Autologous ex vivo gene therapy in which the } \\
\text { COL7A1 gene in inserted into skin cells }\end{array}$ \\
\hline $\begin{array}{l}\text { ABO-101/Abeona } \\
\text { Therapeutics }\end{array}$ & $\begin{array}{l}\text { Mucopolysaccharidosis } \\
\text { IIIB }\end{array}$ & $\begin{array}{l}\text { Gene therapy with recombinant AAV9 expressing } \\
\text { human alpha-N-acetylglucosaminidase }\end{array}$ \\
\hline Cemiplimab/Regeneron & $\begin{array}{l}\text { Skin cancer: squamous } \\
\text { cell carcinoma }\end{array}$ & Fully human monoclonal antibody against PD1 \\
\hline
\end{tabular}
deno-associated virus. Source: BioMedTracker, a service of Sagient Research (http://www.biomedtracker.com)

Laura DeFrancesco is Senior Editor at Nature Biotechnology. myelogenous leukemia was followed two months later by another: Gilead/Kite's Yescarta for diffuse large B-cell lymphomas. Several gene therapies attained breakthrough drug designation; the first AAV gene therapy approval for a retinal disorder looks likely before year's end.

\begin{tabular}{|c|c|c|c|}
\hline \multicolumn{4}{|c|}{ Notable regulatory setbacks ( $3 Q 17)$} \\
\hline Drug/company & \multicolumn{2}{|c|}{ Indication } & Drug information \\
\hline $\begin{array}{l}\text { Evenity (romosozumab) } \\
\text { Amgen }\end{array}$ & \multicolumn{2}{|c|}{$\begin{array}{l}\text { / Osteoporosis/osteo- } \\
\text { penia }\end{array}$} & $\begin{array}{l}\text { 7/16/2017 FDA issued a complete response letter for } \\
\text { this humanized mAb against sclerostin, requesting } \\
\text { data from other clinical trials }\end{array}$ \\
\hline Suptavumab/Regeneror & \multicolumn{2}{|c|}{$\begin{array}{l}\text { Respiratory syncytial } \\
\text { virus }\end{array}$} & $\begin{array}{l}\text { 8/14/2017 Company suspended phase } 3 \text { trial in } \\
\text { preterm infants of fully human mAb against the fusion } \\
\text { protein of RSV due to lack of efficacy }\end{array}$ \\
\hline Sirukumab/J\&J & \multicolumn{2}{|c|}{ Rheumatoid arthritis } & $\begin{array}{l}\text { 9/22/2017 FDA issued a complete response letter } \\
\text { for this humanized mAb against IL- } 6 \text { due to safety } \\
\text { concerns and a negative advisory opinion }\end{array}$ \\
\hline Intepirdine/Axovant & \multicolumn{2}{|c|}{ Alzheimer's disease } & $\begin{array}{l}\text { 9/26/2017 Company suspended phase } 3 \text { trial of } \\
\text { small-molecule selective } 5-\mathrm{HT} 6 \text { receptor antagonist } \\
\text { due to lack of improvement in cognition or in daily } \\
\text { living activity }\end{array}$ \\
\hline $\begin{array}{l}\text { Translarna/PTC } \\
\text { Therapeutics }\end{array}$ & \multicolumn{2}{|c|}{$\begin{array}{l}\text { Duchenne's muscular } \\
\text { dystrophy }\end{array}$} & $\begin{array}{l}11 / 02 / 2017 \text { FDA sent a complete response letter for } \\
\text { this small-molecule exon-skipping drug }\end{array}$ \\
\hline \multicolumn{4}{|c|}{$\begin{array}{l}\text { FDA, US Food and Drug Administration; mAb, monoclonal antibody; J\&J, Johnson and Johnson. Source: } \\
\text { BioMedTracker, a service of Sagient Research (http://www.biomedtracker.com) }\end{array}$} \\
\hline \multicolumn{4}{|c|}{ Notable clinical trial results $(3 Q 17)$} \\
\hline Drug/company In & Indication & \multicolumn{2}{|c|}{ Summary } \\
\hline Emicizumab/Roche $\mathrm{H}$ & Hemophilia A & \multicolumn{2}{|c|}{$\begin{array}{l}\text { 7/10/2017 In phase } 3 \text { randomized study, bispecific antibody } \\
\text { against factor IXa and factor X reduced bleeding episodes by } \\
87 \% \text { compared with blocking agents. (N. Engl. J. Med. } \mathbf{3 7 7} \text {, } \\
809-818,2017 \text { ) }\end{array}$} \\
\hline $\begin{array}{l}\text { Translarna/PTC } \\
\text { Therapeutics }\end{array}$ & $\begin{array}{l}\text { Duchenne's } \\
\text { muscular dys- } \\
\text { trophy }\end{array}$ & \multicolumn{2}{|c|}{$\begin{array}{l}7 / 17 / 2017 \text { In phase } 3 \text { randomized placebo controlled trial, this } \\
\text { small-molecule exon-skipping drug improved six-minute walk } \\
\text { distance in a subgroup compared to placebo. (Lancet } 390 \text {, } \\
1489-1498,2017 \text { ) }\end{array}$} \\
\hline $\begin{array}{l}\text { Luxturna (AAV2. } \\
\text { hRPE65v2)/Spark } \\
\text { Therapeutics }\end{array}$ & $\begin{array}{l}\text { Leber's congeni- } \\
\text { tal amaurosis }\end{array}$ & \multicolumn{2}{|c|}{$\begin{array}{l}\text { 7/13/2017 In open label randomized phase } 3 \text { trial of gene } \\
\text { therapy of human retinal pigment epithelium } 65-k D \text { protein, } \\
\text { vision, light sensitivity and visual field was improved at one } \\
\text { year. (Lancet } 390,849-860,2017 \text { ) }\end{array}$} \\
\hline $\begin{array}{ll}\text { Anacetrapib/Merck } & \text { D } \\
& \text { hy } \\
& \text { ol }\end{array}$ & $\begin{array}{l}\text { Dyslipidemia/ } \\
\text { hypercholester- } \\
\text { olemia }\end{array}$ & \multicolumn{2}{|c|}{$\begin{array}{l}8 / 29 / 2017 \text { In phase } 3 \text { randomized, placebo-controlled trial of } \\
\text { this cholesteryl ester transfer protein inhibitor, the mean level } \\
\text { of non-HDL cholesterol was reduced by } 18 \% \text { and } \mathrm{HDL} \text { choles- } \\
\text { terol level increased by } 104 \% \text {, but major coronary events were } \\
\text { only reduced by } 9 \% \text {. ( } N \text {. Engl. J. Med. } 377,217-1227,2017 \text { ) }\end{array}$} \\
\hline
\end{tabular}

\begin{tabular}{|c|c|c|}
\hline Drug/company & Indication & Summary \\
\hline Ozenoxacin/Medimetriks & $\begin{array}{l}\text { Skin and skin-structure } \\
\text { infections }\end{array}$ & $\begin{array}{l}\text { 11/30/2017 FDA PDUFA date for this novel non- } \\
\text { fluorinated quinolone antibacterial agent }\end{array}$ \\
\hline LR769/LFB Group & Hemophilia A and B & $\begin{array}{l}\text { 11/22/2017 FDA PDUFA date for recombinant } \\
\text { FVIla, produced in the milk of transgenic rabbits to } \\
\text { control bleeding in patients with inhibitors to coagu- } \\
\text { lation factors }\end{array}$ \\
\hline $\begin{array}{l}\text { Andexxa (andexanet } \\
\text { alfa)/Portola }\end{array}$ & Drug toxicity & $\begin{array}{l}\text { 11/30/2017 EMA CHMP decision on universal } \\
\text { Factor Xa inhibitor antidote to address uncontrol- } \\
\text { lable bleeding }\end{array}$ \\
\hline $\begin{array}{l}\text { Luxturna/Spark } \\
\text { Therapeutics }\end{array}$ & $\begin{array}{l}\text { Leber's congenital } \\
\text { amaurosis }\end{array}$ & $\begin{array}{l}\text { 1/12/2018 FDA PDUFA date for this gene therapy } \\
\text { of AAV-delivered human retinal pigment epithelium } \\
65-k D \text { protein }\end{array}$ \\
\hline $\begin{array}{l}\text { Macrilen (growth hor- } \\
\text { mone secretagogue)/ } \\
\text { Aeterna }\end{array}$ & Short stature & $\begin{array}{l}\text { 12/29/2017 FDA 2nd PDUFA date ghrelin receptor } \\
\text { targeting growth hormone secretagogue }\end{array}$ \\
\hline $\begin{array}{l}\text { Benralizumab/ } \\
\text { AstraZeneca }\end{array}$ & Asthma & $\begin{array}{l}\text { 12/31/2017 FDA PDUFA date for this humanized } \\
\text { monoclonal antibody against IL-5R } \alpha\end{array}$ \\
\hline $\begin{array}{l}\text { Ibalizumab/ } \\
\text { Theratechnologies }\end{array}$ & HIV/AIDS & $\begin{array}{l}\text { 1/3/2018 FDA PDUFA date for this humanized } \\
\text { monoclonal antibody against CD4, which inhibits } \\
\text { entry of HIV into CD4 cells }\end{array}$ \\
\hline $\begin{array}{l}\text { Lutathera (lutetium Lu } \\
177 \text { dotatate)/Advanced } \\
\text { Accelerator Applications }\end{array}$ & Neuroendocrine tumors & $\begin{array}{l}1 / 26 / 2018 \text { FDA PDUFA data for this radiolabeled } \\
\text { somatostatin }\end{array}$ \\
\hline $\begin{array}{l}\mathrm{VX}-661 \text { (tezacaftor)/ } \\
\text { Vertex }\end{array}$ & Cystic fibrosis & $\begin{array}{l}\text { 2/28/2018 FDA PDUFA date for this small-molecule } \\
\text { CFTR corrector }\end{array}$ \\
\hline Emicizumab/Roche & Hemophilia A & $\begin{array}{l}\text { 2/23/2018 FDA PDUFA date for this bispecific IgG } \\
\text { antibody against factor IXa and factor } X\end{array}$ \\
\hline $\begin{array}{l}\text { Tildrakizumab/Sun } \\
\text { Pharmaceuticals }\end{array}$ & Psoriasis & $\begin{array}{l}\text { 3/1/2018 FDA PDUFDA date for this fully human } \\
\text { monoclonal antibody IgG } 1 \kappa \mathrm{CD} 23 \mathrm{mAb}\end{array}$ \\
\hline
\end{tabular}

